\title{
Treatment for intractable asthma: bronchial thermoplasty
}

\author{
Haruhito Sugiyama*, Motoyasu likura, Satoru Ishii, Masayuki Hojo \\ Department of Respiratory Medicine, National Center for Global Health and Medicine, Tokyo, Japan.
}

\begin{abstract}
Bronchial Thermoplasty (BT) is an epoch-making treatment that reduces bronchial smooth muscle by using a bronchoscope to reach the basket catheter to the bronchus and directing high-frequency current directly into the bronchi. In GINA Guidelines 2019, BT is considered to be additional treatment at Step V (the most severe), and the evidence level is B. The Japanese guidelines (JGL) also added BT as a treatment for Step 4, but it is reserved because there are still unclear points regarding long-term efficacy and safety. In Japan, as of April 1, 2019, 672 treatments were performed at 123 institutions nationwide. The average age of patients was 54.1 years, but 84 cases were over 70 years old. The average value of $\%$ FEV1 was $78.2 \%$, but there were 94 cases less than $60 \%$. There were 32 cases that underwent BT treatment at our institution. Of them, 12 cases with progress up to one year later could be observed. The average age was 56.1 years old, and each of 6 men and women had a $\%$ FEV 1 of $70.5 \%$. One year later, AQLQ and \%FEV1 improved, and the number of exacerbations decreased, but exhaled NO values increased. \%FEV1 improvement might be due to poorer lung function $(70.5 \%$ vs. $77.8 \%)$ and more BT activation (average 1.28 times AIR2) compared to the AIR2 trial. In terms of improvement in \%FEV1, patients with moderate obstructive disorder from 50 to $80 \%$ responded well after BT treatment. In the near future, various new antibody preparations such as IL-4 / 13 antibody and anti-TSLP antibody are expected to be promoted. Therefore, we first consider whether these antibody preparations can be applied to patients with refractory asthma. We consider that BT is indicated only when there is no indication or no expected effect of antibody preparations. In other words, BT treatment is the last resort of intractable asthma, and it is the duty of medical professionals involved in BT treatment to be able to advocate when it is best to give BT to such patients.
\end{abstract}

Keywords: Bronchial thermoplasty, intractable asthma, refractory asthma, bronchial asthma, efficacy, safety

\section{Introduction}

Bronchial asthma is a disease characterized by clinical symptoms such as chronic airway inflammation and variability in airway stenosis (wheezing, dyspnea) and cough (1). The current standard treatment, combination of inhaled corticosteroids (ICS) and long acting $\beta 2$ agonists (LABA), is used to suppress airway inflammation and dilate the airways. Although many patients can be controlled by this treatment method, there are still patients whose symptoms persist, and it is difficult to treat as so-called "refractory asthma".

Whatever the cause of bronchial asthma, the end result is airway remodeling such as subepithelial fibrosis, smooth muscle thickening, and submucosal gland hyperplasia resulting in irreversible airway stenosis and increased airway hyperresponsiveness, it has contributed to intractable asthma. Unfortunately, ICS and LABA are ineffective against airway remodeling once it occurs. In contrast, bronchial thermoplasty has been developed for the purpose of physically suppressing airway contraction.

\section{Bronchial Thermoplasty (BT)}

BT is an epoch-making treatment that reduces bronchial smooth muscle by using a bronchoscope to reach the basket catheter to the bronchus and directing highfrequency current directly into the bronchi $(2,3)$. Although it was listed in Japan since April 2015, there are no clinical trials in Japan, and there is little knowledge about BT.

As a mechanism of action, in basic experiments using animals, BT reduces bronchial smooth muscle mass and suppresses airway hyperresponsiveness (4). In experiments using a tracheal ring, tracheal contraction due to acetylcholine disappears when heat treatment is performed at $60^{\circ} \mathrm{C}$ or higher for 10 seconds (5). Recently, a decrease in airway smooth muscle capacity and nerverelated tissue due to BT has been correlated with clinical effects, and in particular, a decrease in nerve-related tissue due to BT is closely related to suppression of exacerbations (6). Significant reduction of nerve fibers in the submucosa and airway smooth muscle was reported (7). Based on the above, BT is thought to act directly on 
airway smooth muscle and surrounding nerve-related tissues to suppress airway contraction.

In GINA Guidelines 2019, BT is considered to be additional treatment at Step V (the most severe), and the evidence level is B. The Japanese guidelines (JGL) also added BT as a treatment for Step 4, but it is reserved because there are still unclear points regarding long-term efficacy and safety (8).

BT insurance coverage in Japan is for patients with severe asthma over 18 years of age who cannot control asthma symptoms with high-dose ICS and LABA and are candidates for bronchoscopic procedures. In fact, in addition to high doses of ICS and LABA, even using other drugs (long acting anti-cholinergic agents, theophylline preparations, biologic agents, etc.), asthmatic patients who cannot control asthma symptoms and required steroid burst several times a year are targeted patients. In theory, all asthma patients regardless of disease types can be indicated.

BT is basically not contraindicated in patients who can safely undergo bronchoscopy. However, because of the use of high-frequency current, users of implantable medical devices such as pacemakers and ICDs, patients with unstable asthma symptoms, patients with respiratory infections such as bronchiectasis, and blood coagulation disorders are contraindicated. For BT, long-term usefulness and safety exceeding 5 years have not been established. In addition, there is no example of BT used a second time in Europe and the United States, so it is currently a one-time treatment for life.

\section{Evidence for BT}

The most extensive AIR2 study is explained (9). The subjects were bronchial asthma patients aged 18 to 65 years who had been using ICS (beclomethasone equivalent $1000 \mu \mathrm{g} /$ day or more) and LABA (salmeterol equivalent $100 \mu \mathrm{g} /$ day or more) for 4 weeks or more (but oral steroids were $10 \mathrm{mg}$ /day PSL equivalent days or less). Inclusion criterion were the following. The AQLQ score, which is an index of QOL, is 6.25 or less, the $\%$ Forced expiratory volume in 1 second ( $\%$ FEV 1$)$ before administration of bronchodilators is $60 \%$ or more, airway hypersensitivity has been demonstrated in the methacholine inhalation test, and asthma symptoms at least 2 days during the 4-week observation period. It was also necessary to be a non-smoker (no smoking during the last year, less than 10 pack-years). However, patients with chronic sinus disease or emphysema, and patients who were hospitalized for more than 3 asthma attacks or lower respiratory tract infections in the previous year were excluded. In a randomized, double blind study, 190 were assigned to the BT group and 98 to the Sham group (with bronchial catheters inserted but not energized). The average age of patients in the BT group/ Sham group was 40.7 years $/ 40.6$ years old, and the $\%$ FEV1 was $77.8 \% / 79.7 \%$, which were relatively good.
The primary endpoint was the degree of improvement in AQLQ. As a result, the rate of clear improvement in AQLQ $(+0.5$ or higher) was $80.9 \%$ in the BT group, $63.2 \%$ in the Sham group, and clear exacerbation $(-0.5$ or higher) were $2.9 \%$ in the BT group and $7.4 \%$ in the Sham group, with statistically significant differences between the two groups. In addition, the number of serious asthma exacerbations and emergency visits, which were secondary endpoints, were clearly lower in the BT group. However, there was no difference between the two groups in Peak flow rate (PEF) values and FEV1. Adverse reactions included exacerbation of asthma, wheezing, dyspnea, chest pain, lower respiratory tract infection, atelectasis, and blood clots, but no serious events were observed.

The above is the result for 1 year after BT treatment, but after that, the results for 5 years after BT treatment were reported (10). Both the serious exacerbation of asthma and a decrease in the number of emergency visits observed in the AIR2 study after 1 year of BT treatment were confirmed even after 5 years of BT treatment. The $\%$ FEV1 did not change before and after inhalation of bronchodilators. Recently, a 3 years post-marketing survey (PAS2) in the US was reported (11), as in AIR2, over 1 to 3 years after BT, severe asthma exacerbations, emergency visits, and number of hospitalizations were clearly decreased in the group.

\section{Current status of BT in Japan}

As of April 1, 2019, 672 treatments were performed at 123 institutions nationwide. The average age of the patients was 54.1 years, but 84 cases were over 70 years old. The average value of $\%$ FEV1 was $78.2 \%$, but there were 94 cases less than $60 \%$. As anesthesia methods, local anesthesia was often performed at 91 institutions (76\%) (information provided by Boston Scientific Japan). In the real world, older and more severe cases are treated with BT. We have 32 BT treatments. Here are our champion cases and problem cases.

Case 1 is a 62-year-old male with a morbidity of 18 years and a history of smoking of 20 pack-years. Although oral steroids are not commonly used, oral administration of steroids is required 5-8 times a year, and severe asthma corresponding to JGL Step 4 and GINA Step V. The drugs used were Symbicort ${ }^{\circledR} 8$ inhalations/day, tiotropium $5 \mu \mathrm{g} /$ day, theophylline 400 $\mathrm{mg}$ /day, montelukast $10 \mathrm{mg} /$ day, and omalizumab was canceled due to ineffectiveness. When this patient was treated with BT, unlike the AIR2 study, improvement in respiratory function and decrease in exhaled NO (FeNO) levels were observed (Figure 1). Figure 2 and Figure 3 show marked improvement of respiratory function and impedance. Complications, wheezing, chest pain and chest discomfort were observed immediately after BT treatment, and chest radiographs showed transient atelectasis and infiltrative shadows consistent with the 


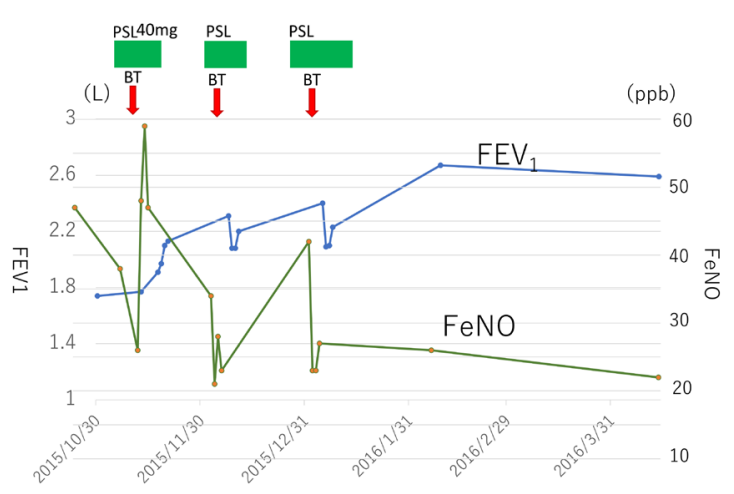

Figure 1. Changes of FEV1 and FeNO in Case 1. FEV1, forced expiratory volume in 1 second; FeNO, fractional exhaled nitric oxide; BT, Bronchial Thermoplasty.
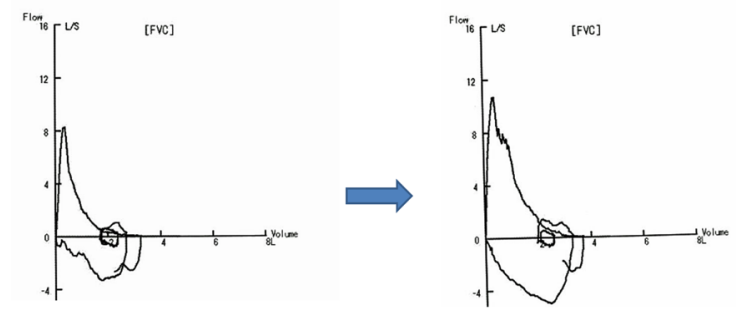

FVC 3.26 L, \%FVC 90.8\%, FEV 1.77 L, \%FEV $159.6 \%$ $\mathrm{FEV}_{1} \% 54.3 \%$ $\mathrm{V}_{50} 0.58 \mathrm{~L} / \mathrm{s}(15.3 \%)$ $\mathrm{V}_{25} 0.16 \mathrm{~L} / \mathrm{s}(11.1 \%)$ $\mathrm{V}_{25} 0.16 \mathrm{~L} / \mathrm{s}(11.39$
$\mathrm{RV} / \mathrm{TLC} 37.4 \%$ FEV 12.46 L, \%FEV $184.5 \%$ FEV $1 \% 65.8 \%$ $\mathrm{V}_{50} 3.70 \mathrm{~L} / \mathrm{s}(47.3 \%)$ $\mathrm{V}_{50} 3.70 \mathrm{~L} / \mathrm{s}(47.3 \%)$
$\mathrm{V}_{25} 1.39 \mathrm{~L} / \mathrm{s}(27.3 \%)$ $\mathrm{RV} / \mathrm{TLC} 34.3 \%$

Figure 2. Flow-volume curve after 1 year in Case 1. BT, Bronchial Thermoplasty.
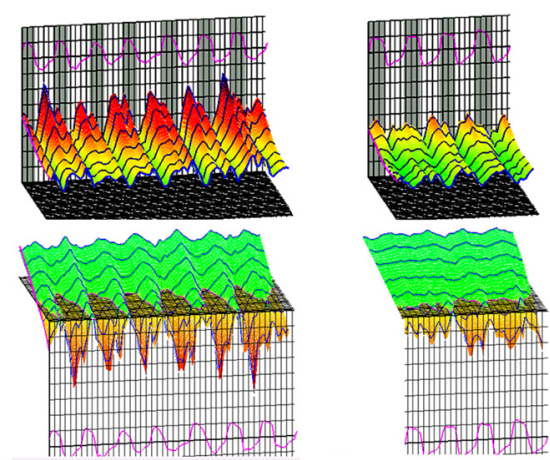

Figure 3. Change of respiratory impedance before (left side) and after (right side) BT in Case 1. BT, Bronchial Thermoplasty.

BT site. These phenomena are common in all cases and appear immediately after BT treatment and disappear 5-7 days later. Chest high-resolution computed tomography (HRCT) shows marked thickening of the bronchial wall involving the surrounding bronchial tissue and severe narrowing of the bronchial lumen (Figure 4). It is speculated that extensive thermal damage has spread to and around the bronchi due to BT treatment.

Case 2 is a 33-year-old, non-smoker woman who suffered for 21 years. Previously she was steroid dependent but it was possible to discontinue steroids

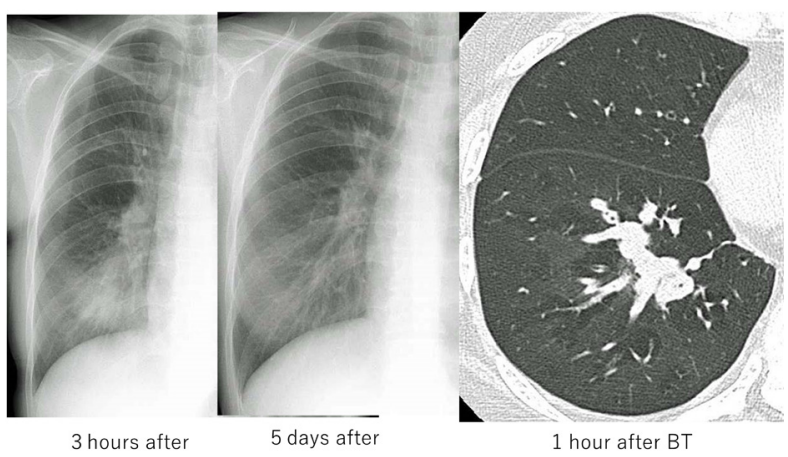

Figure 4. Radiological changes after $1^{\text {st }}$ BT. BT, Bronchial Thermoplasty; HRCT, high-resolution computed tomography.

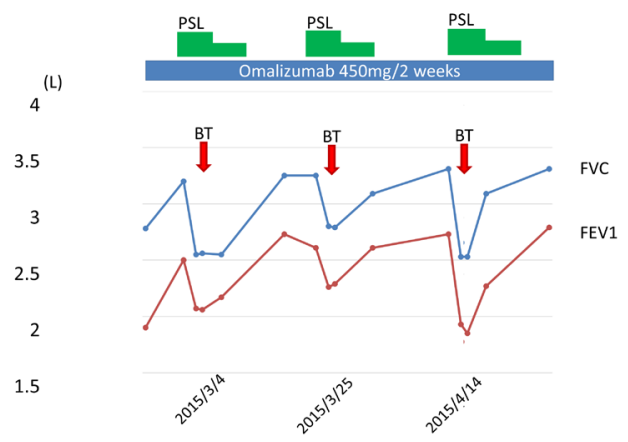

Figure 5. Changes of FVC and FEV1 after BT in the Case 2. FVC, forced vital capacity; FEV1, forced expiratory volume in 1 second; BT, Bronchial Thermoplasty.

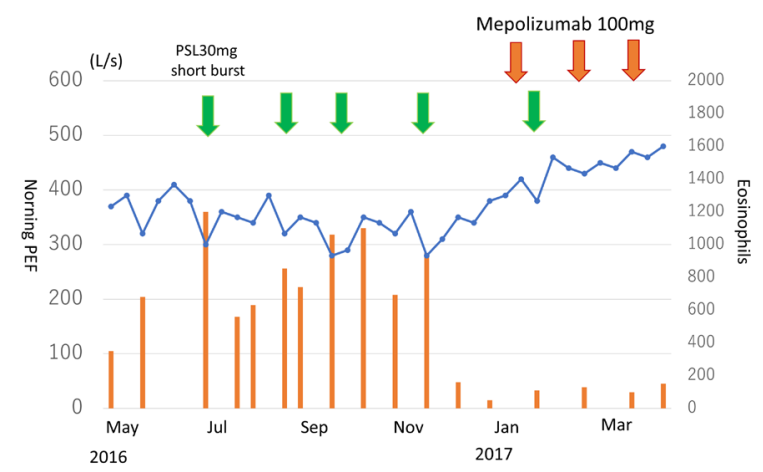

Figure 6. Clinical course 1 to 2 years after BT in the Case 2. BT, Bronchial Thermoplasty.

after administration of omalizumab. The number of exacerbations of asthma within the past year has been 3, and severe asthma corresponding to JGL Step 4 and GINA Step V. The drugs used were Relvar $200^{\circledR}$ inhalations/ day, theophylline $400 \mathrm{mg}$ /day, montelukast $10 \mathrm{mg}$ /day, omalizumab $450 \mathrm{mg} / 2$ weeks. After BT treatment, the morning PEF value increased from 350 to $500 \mathrm{~L} / \mathrm{S}$, and the \%FEV1 also improved markedly from $65.5 \%$ to $80.1 \%$ (Figure 5). However, after 2 months of BT treatment, the PEF value and FEV1 decreased gradually, and a steroid burst was required once a month (Figure 6). 
At the same time, the number of eosinophils in the blood increased, and chest CT showed infiltrative shadows. Therefore, when mepolizumab was introduced because it was considered as eosinophilic pneumonia, improvement of asthma symptoms, disappearance of chest infiltration shadows, and improvement of respiratory function were observed (Figure 6). In this case, omalizumab was originally introduced but it was insufficiently controlled, so there was a history so that BT treatment was introduced. Eosinophilia was not clear before BT introduction. However, although the respiratory function was once improved by the introduction of BT, the basic immunological abnormality was not controlled, and it is considered that the asthma symptoms worsened due to the exacerbation of eosinophilic inflammation.

Table 1, Figure 7 and Figure 8 show the results of 12 cases in which BT was performed at our hospital and the

Table 1. Outcomes of BT in National Center for Global Health and Medicine $(n=12)$

\begin{tabular}{lccc}
\hline Items & Before & $1 \mathrm{M}$ after BT & $12 \mathrm{M}$ after BT \\
\hline AQLQ & $4.9+1.1$ & $5.7+1.4^{*}$ & $5.8+1.3^{*}$ \\
ACQ-5 & $1.5+0.9$ & $0.9+1.0^{*}$ & $0.9+0.9^{*}$ \\
ACT & $19.0+4.2$ & & $20.5+4.4$ \\
\%FEV1 & $70.5+21.7$ & $82.2+20.6^{*}$ & $82.3+21.8^{* *}$ \\
FeNO & $50.2+70.8$ & $51.3+61.0$ & $68.8+80.4^{*}$ \\
Exacerbation & $5.8+5.3$ & & $2.0+2.8^{*}$ \\
\hline
\end{tabular}

*: $P<0.05 ; * *: P<0.01$.
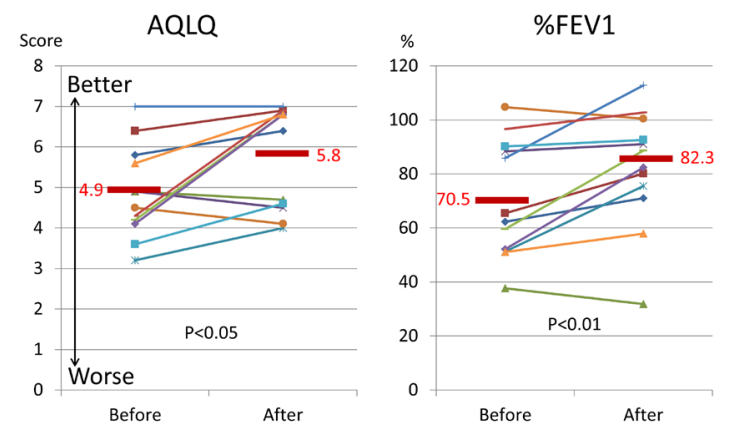

Figure 7. Change of AQLQ and \%FEV1 $12 \mathrm{mo}$ after BT. FEV1, forced expiratory volume in 1 second; BT, Bronchial Thermoplasty.
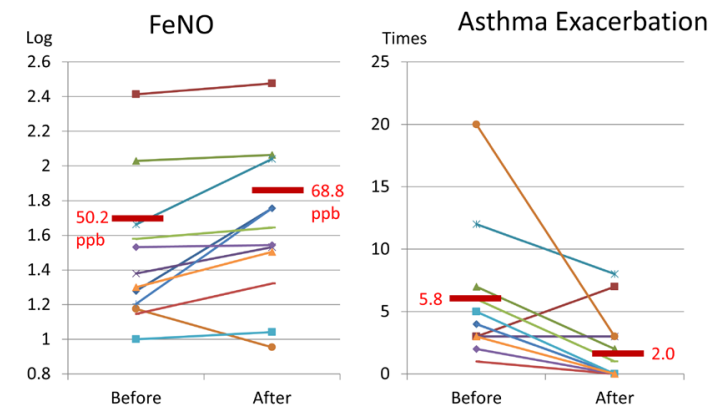

Figure 8. Change of FeNO and asthma exacerbation 12 months after BT. FeNO, fractional exhaled nitric oxide; BT, Bronchial Thermoplasty. progress up to one year later could be observed (12). The average age was 56.1 years old, and each of 6 men and women had a $\%$ FEV1 of $70.5 \%$. One year later, AQLQ and $\%$ FEV1 improved, and the number of exacerbations decreased, but exhaled NO value increased. \%FEV1 improvement might be due to poorer lung function (70.5\% vs. 77.8\%) and more BT activations (average 1.28 times of AIR2) compred to the AIR2 trial. In terms of improvement in \%FEV 1 , patients with moderate obstructive disorder from 50 to $80 \%$ responded well after BT treatment (Figure 7). In addition, the absence of a decrease in exhaled NO level is proof that it has no effect on eosinophilic inflammation of the airway in the background. We reported dilation of the bronchial lumen and decreased bronchial wall thickness was evident after BT using three-dimensional airway analysis (13). Recently, Ishii et al. have reported chest HRCT at inspiratory and expiratory levels before and after BT treatment, and reported that the expiratory lung volume decreases after BT treatment (Figure 9) (14). Langton et al. also reported increase of the luminal airway volume and decrease of residual volume after BT $(15,10)$. It can be said that measurement of expiratory lung volume can be a tool for estimating the usefulness of BT treatment. Langston et al. also reported that BT improved gas trapping and speculated this improvement may relate to changes in the mechanical properties of small airways that are not measured with spirometry (17).

Concerning severe adverse effects, we experienced a case of Aspergillus and Nocardia infections after BT (18). There were two cases of pulmonary cysts/pneumothorax (19), and other cases of hemoptysis as serious adverse reactions associated with the BT treatment we performed. Regarding hemoptysis, there are reports in the literature that mediastinal hemorrhage and hemothorax occurred several days after BT treatment, and the cause was pseudo bronchial aneurysm, which was improved by bronchial embolization (20). In the aforementioned AIR2 test, there was a case of hemoptysis and bronchial artery embolization. The association between hemoptysis and BT has not been proven, but BT treatment can cause rare but severe bleeding.

\section{Future task of BT}

Recently, antibody therapies targeting IL-5 (mepolizumab, benralizumab) have appeared and new tools have been added to treat asthma. The problem here is which should be prioritized, the antibody formulations or BT treatment. At present, it is reasonable to start with antibody preparations first for patients with appropriate indications. This is because BT treatment is highly invasive for patients and cannot control airway inflammation behind asthma. The introduction of BT treatment is appropriate for cases in which various antibody preparations are still insufficiently controlled. Recently, we have experienced a case in which the 

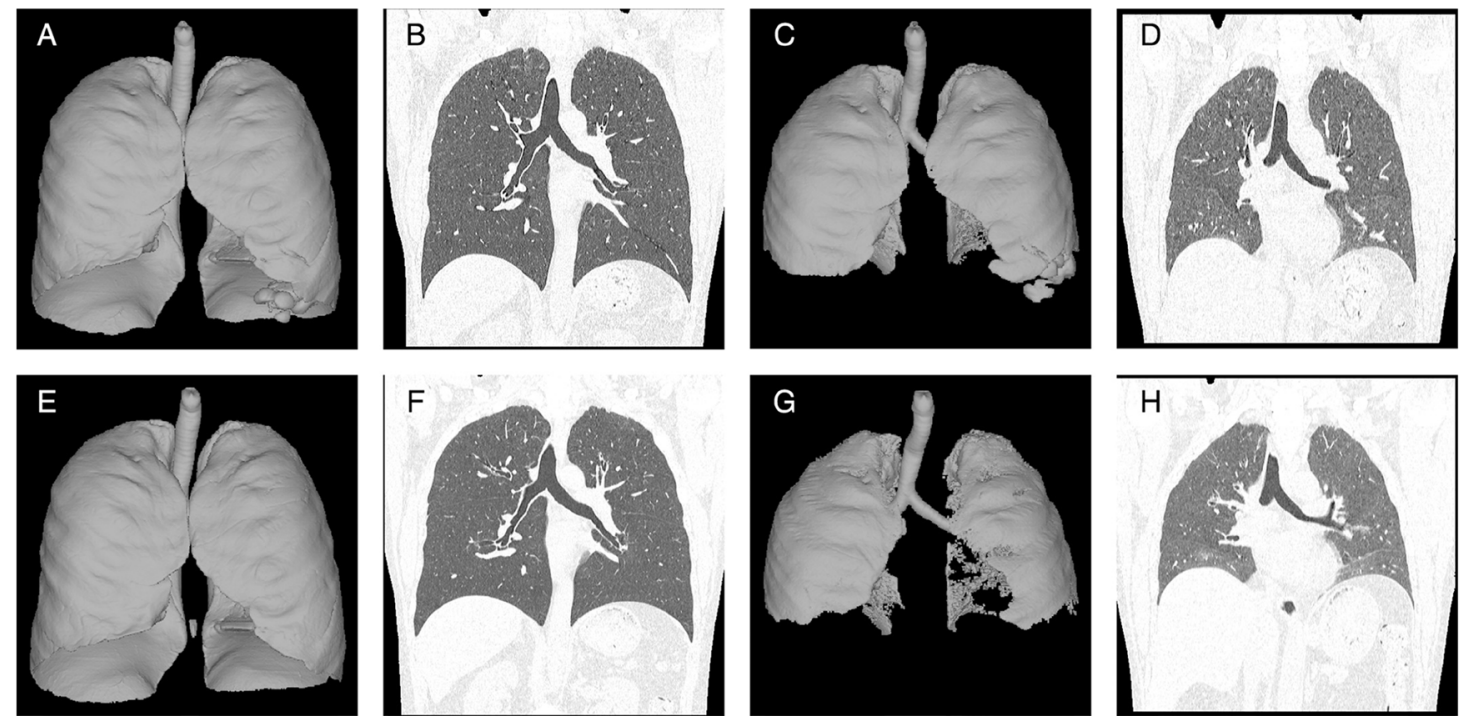

Figure 9. Lung capacity by virtual place in the inspiration and expiration. Upper row: Before BT; Lower row: After BT. BT, Bronchial Thermoplasty.

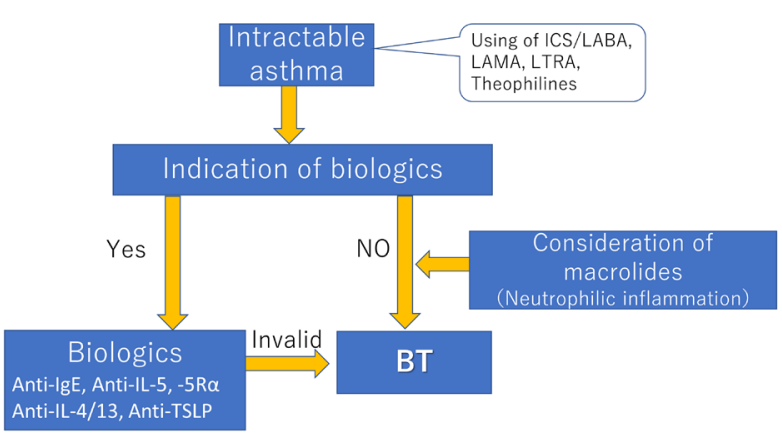

Figure 10. Flow chart in the decision making of BT. BT, Bronchial Thermoplasty.

amount of goblet cells in the bronchial mucosa decreased and the amount of sputum decreased after BT treatment (21). BT treatment may be effective even in cases with much sputum and possible involvement of neutrophilic inflammation.

In the near future, various new antibody preparations such as IL-4/13 antibody and anti-TSLP antibody are expected to be promoted. Therefore, we first consider whether these antibody preparations can be applied to patients with refractory asthma. We consider that BT is a good indication only when there is no indication or no expected effect of antibody preparations. In other words, BT treatment is the last resort of intractable asthma. Figure 10 shows a flowchart of antibody formulation and BT treatment, which we consider at this time. However, it should be emphasized that this flowchart only describes the principle, since BT treatment may be prioritized due to the individual circumstances of each patient.

The biggest problem in conducting BT treatment is that its effectiveness cannot be predicted in advance. Since BT is a highly invasive treatment, if any factor that

can predict effectiveness can be identified in advance, unnecessary treatment should be avoided. Since BT is a one-time treatment at present, we believe that it is the duty of medical professionals involved in BT treatment to be able to advocate when it is best to give BT to such patients.

\section{References}

1. Tamaoki J. Asthma Prevention and management Guideline 2018 in Japan. Arerugi. 2018; 67:1263-1268. (in Japanese)

2. Cox G, Miller JD, McWilliams A, Fitzgerald JM, Lam S. Bronchial Thermoplasty for Asthma. Am J Respir Crit Care Med. 2006; 173:965-969.

3. Cox G, Thomson NC, Rubin AS, Niven RM, Corris PA, Siersted HC, Olivenstein R, Pavord ID, McCormack D, Chaudhuri R, Miller JD, Laviolette M; AIR Trial Study Group. Asthma control during the year after bronchial thermoplasty. N Engl J Med. 2007; 356:1327-1337.

4. Danek CJ, Lombard CM, Dungworth DL, Cox PG, Miller JD, Biggs MJ, Keast TM, Loomas BE, Wizeman WJ, Hogg JC, Leff AR. Reduction in airway hyperresponsiveness to methacholine by the application of RF energy in dogs. J Appl Physiol (1985). 2004; 97:19461953.

5. Dydra P, Tazzeo T, DoHarris L, Nilius B, Roman HN, Lauzon AM, Aziz T, Lukic D, Janssen LJ. Acute response of airway muscle to extreme temperature includes disruption of actin-myosin interaction. Am J Respir Cell Mol Biol. 2011; 44:213-221.

6. Pretolani M, Bergqvist A, Thabut G, Dombret MC, Knapp D, Hamidi F, Alavoine L, Taillé C, Chanez P, Erjefält JS, Aubier M. Effectiveness of bronchial thermoplasty in patients with severe refractory asthma: Clinical and histopathologic correlations. J Allergy Clin Immunol. 2017; 139:1176-1185.

7. Facciolongo N, Di Stefano A, Pietrini V, Galeone C, Bellanova F, Menzella F, Scichilone N, Piro R, Bajocchi GL, Balbi B, Agostini L, Salsi PP, Formisano D, Lusuardi 
M. Nerve ablation after bronchial thermoplasty and sustained improvement in severe asthma. BMC Pulm Med. 2018; 18:29.

8. Ichinose M, Sugiura H, Nagase H, Yamaguchi M, Inoue H, Sagara H, Tamaoki J, Tohda Y, Munakata M, Yamauchi K, Ohta K; Japanese Society of Allergology. Japanese guidelines for adult asthma 2017. Allergol Int. 2017; 66:163-189.

9. Castro M, Rubin AS, Laviolette M, et al. Effectiveness and Safety of Bronchial Thermoplasty in the Treatment of Severe Asthma. Am J Respir Crit Care Med. 2010; 181:116-124.

10. Wechsler ME, Laviolette M, Rubin AS, et al. Bronchial thermoplasty: Long-term safety and effectiveness in patients with severe persistent asthma. J Allergy Clin Immunol. 2013; 132:1295-1302.

11. Chupp G, Laviolette M, Cohn L, McEvoy C, Bansal S, Shifren A, Khatri S, Grubb GM, McMullen E, Strauven R, Kline JN; Other members of the PAS2 Study Group. Long-term outcomes of bronchial thermoplasty in subjects with severe asthma: a comparison of 3-year follow-up results from two prospective multicenter studies. Eur Respir J. 2017; 50: pii:1700017.

12. Iikura M, Hojo M, Nagano N, Sakamoto K, Kobayashi K, Yamamoto S, Hashimoto M, Ishii S, Izumi S, Sugiyama H. Bronchial thermoplasty for severe uncontrolled asthma in Japan. Allergol Int. 2018; 67:273-275.

13. Ishii S, Iikura M, Hojo M, Sugiyama H. Use of 3DCT airway analysis software to assess a patient with severe persistent bronchial asthma treated with bronchial thermoplasty. Allergol Int. 2017; 66:501-503.

14. Ishii S, Iikura M, Shimoda Y, Izumi S, Hojo M, Sugiyama H. Evaluation of expiratory capacity with severe asthma following bronchial thermoplasty. Respirol Case Rep. 2018; 7:e00387.

15. Langton D, Sloan G, Banks C, Bennetts K, Plummer V,
Thien F. Bronchial thermoplasty increases airway volume measured by functional respiratory imaging. Respir Res. 2019; 20:157.

16. Langton D, Ing A, Sha J, Bennetts K, Hersch N, Kwok M, Plummer V, Thien F, Farah C. Measuring the effects of bronchial thermoplasty using oscillometry. Respirology. 2019; 24:431-436.

17. Langton D, Ing A, Bennetts K, Wang W, Farah C, Peters M, Plummer V, Thien F. Bronchial thermoplasty reduces gas trapping in severe asthma. BMC Pulm Med. 2018; 18:155.

18. Matsubayashi S, Iikura M, Numata T, Izumi S, Sugiyama H. A case of Aspergillus and Nocardia infections after bronchial thermoplasty. Respirol Case Rep. 2018; 7:e00392.

19. Funatsu A, Kobayashi K, Iikura M, Ishii S, Izumi S, Sugiyama H. A case of pulmonary cyst and pneumothorax after bronchial thermoplasty. Respirol Case Rep. 2017; 6: $\mathrm{e} 00286$.

20. Nguyen DV, Murin S. Bronchial artery pseudoaneurysm with major hemorrhage after bronchial thermoplasty. Chest. 2016; 149: e95-97.

21. Nagano N, Iikura M, Ito A, Miyawaki E, Hashimoto M, Sugiyama H. Bronchial thermoplasty for severe asthma with mucus hypersecretion. Intern Med. 2019; 58:16131616.

Received September 4, 2019; Revised November 18, 2019; Accepted November 25, 2019.

*Address correspondence to:

Haruhito Sugiyama, Department of Respiratory Medicine, National Center for Global Health and Medicine, 1-21-1 Toyama Shinjuku-ku, Tokyo 162-8655, Japan.

E-mail: hasugiya@hosp.ncgm.go.jp 